\title{
EVERY MAPPING OF THE PSEUDO-ARC ONTO ITSELF IS A NEAR HOMEOMORPHISM
}

\author{
MICHEL SMITH
}

\begin{abstract}
The theorem stated in the title is proven. It follows then that the homeomorphism group of the pseudo-arc is dense in the space of continuous functions from the pseudo-arc onto itself.
\end{abstract}

In general, little is known about the structure of the space of homeomorphisms of a space onto itself. Beverly Brechner $[\mathbf{B r 1}, \mathbf{B r 2}]$ and Wayne Lewis $[\mathbf{L w}]$ have published some results concerning the homeomorphism group of the pseudo-arc. We show that the homeomorphism group of the pseudo-arc is dense in the space of continuous functions from the pseudo-arc onto the pseudo-arc.

A continuum is a compact connected metric space. The pseudo-arc is a hereditarily indecomposable chainable continuum. For the definition and properties of crookedness the reader should consult Bing $[\mathbf{B}]$. Theorems A and B are due to Bing [B].

Definition. Suppose that $X$ and $Y$ are continua and $f: X \rightarrow Y$ is continuous. Then $f$ is said to be a near homeomorphism if it is true that for each positive number $\varepsilon$ there exists a homeomorphism $h: X \rightarrow Y$ so that $d(f(x), h(x))<\varepsilon$ for all $x \in X$.

The theorem stated in the title follows from Theorem 1.

DEFinition. Suppose that $N$ is a function from the set of positive integers $\{1, \ldots, r\}$ into the set of positive integers $\{1, \ldots, s\}$. Then $N$ is a pattern means that for each $1 \leq i<r,|N(i+1)-N(i)| \leq 1$.

DEfinition. If $N$ is a pattern then the chain $D$ follows the pattern $N$ in the chain $C$ means that if $D=d_{1}, \ldots, d_{r}$, and $C=c_{1}, \ldots, c_{s}$, then $N:\{1, \ldots, r\} \rightarrow$ $\{1, \ldots, s\}$ and $d_{i} \subset c_{N(i)}$.

DEFINITION. The chain $E$ is a consolidation of the chain $D$ if each link of $E$ is the union of a subcollection of $D$ and each link of $D$ is a subset of a link of $E$.

DEFINITION. The chain $C$ is a chain from the point $P$ to the point $Q$ means that $P$ belongs to an end link of $C$ and to no other link of $C$ and $Q$ belongs to the other end link of $C$ and to no other link.

Notation. If $H$ and $K$ are sets then let

$$
d(H, K)=\operatorname{glb}\{d(x, y) \mid x \in H, y \in K\} .
$$

TheOREM A (BING [B]). Suppose that $N:\{1, \ldots, n\} \rightarrow\{1, \ldots, r\}$ is a pattern, $N$ is onto, $N(1)=1, N(n)=r, D_{1}, D_{2}, \ldots$ is a sequence of chains from the point $P$ to the point $Q$ such that $D_{1}$ has $r$ links, $D_{i+1}$ is crooked in $D_{i}$ for each positive

Received by the editors January 14, 1983 and, in revised form, August 22, 1983. Presented to the Society, November 12-13, 1982 in Baton Rouge, Louisiana.

1980 Mathematics Subject Classification. Primary 54F20.

Key words and phrases. Pseudo-arc, near homeomorphism, homeomorphism group. 
integer $i$, and $\operatorname{mesh}\left(D_{i}\right) \leq 1 / i$. Then there is an integer $j$ and a chain $E$ from $P$ to $Q$ such that $E$ is a consolidation of the chain $D_{j}$ and $E$ follows pattern $N$ in $D_{1}$.

THEOREM B (BING $[\mathbf{B}])$. Suppose that $M_{n}(n=1,2)$ is a compact closed set; $\varepsilon_{1}, \varepsilon_{2}, \ldots$ is a sequence of positive numbers with a finite sum; and $X_{n, 1}, X_{n, 2}, \ldots$ is a sequence of chains such that for each positive integer $i$, (1) $X_{n, i}$ covers $M_{n}$, (2) each element of $X_{n, i}$ intersects $M_{n}$, (3) no element of $X_{n, i}$ has a diameter of more than $\varepsilon_{i}$, and (4) if the $j$ th element of $X_{n, i+1}$ intersects the kth element of $X_{n, i}$, then the distance between the jth element of $X_{m, i+1}(m=1,2)$ and the kth element of $X_{m, i}$ is less than $\varepsilon_{i}$. Then there is a homeomorphism $T$ carrying $M_{1}$ into $\mathrm{M}_{2}$.

LEMMA. If $X$ and $\tilde{X}$ are indecomposable continua and $f$ is a map from $X$ onto $\tilde{X}$ then there exist two points $a$ and $b$ so that $a$ and $b$ are in different composants of $X$ and $f(a)$ and $f(b)$ are in different composants of $\tilde{X}$.

P.ROOF. Let $P$ and $Q$ be two points of $\tilde{X}$ that lie in different composants of $\tilde{X}$. Then if $f^{-1}(P)$ and $f^{-1}(Q)$ are both subsets of the same composant $C$ of $X$, let $R$ be a point of $X$ lying in a composant of $X$ different from $C$. Then $R$ and $f^{-1}(Q)$ are in different composants of $X$ and $f(R)$ lies in a composant which either does not contain $Q$ or does not contain $P$. Assume the former, then let $a=R$ and $b \in f^{-1}(Q)$. In the latter case let $a=R$ and $b \in f^{-1}(P)$.

THEOREM 1. Suppose that $X$ and $\tilde{X}$ are pseudo-arcs, $f: X \rightarrow \tilde{X}$ is a map of $X$ onto $\tilde{X}$ and $\varepsilon>0$. Then there exists a homeomorphism $h: X \rightarrow \tilde{X}$ such that if $x \in X$ then $d(h(x), f(x))<\varepsilon$.

Proof. Let $P$ and $Q$ be two points of $X$ so that $P$ and $Q$ lie in different composants of $X$ and $f(P)$ and $f(Q)$ lie in different composants of $\tilde{X}$. Let $0<$ $\delta_{1}<\varepsilon / 4$.

Let $C^{1}, C^{2}, \ldots$ be a sequence of chains from $f(P)$ to $f(Q)$ so that for each positive integer $i, C^{i+1}$ is crooked in $C^{i}, \operatorname{mesh}\left(C^{i}\right)<1 / i$, the closure of each link of $C^{i+1}$ is a subset of a link of $C^{i}$, the closures of two links of $C^{i}$ intersect if and only if the links intersect, and so that $C^{1}$ is a $\delta_{1}$ chain.

Let $D^{1}, D^{2}, \ldots$ be a sequence of chains from $P$ to $Q$ so that for each positive integer $i, D^{i+1}$ is crooked in $D^{i}, \operatorname{mesh}\left(D^{i}\right)<1 / i$, the closure of each link of $D^{i+1}$ is a subset of a link of $D^{i}$, the closures of two links of $D^{i}$ intersect if and only if the links intersect, and so that if $d \in D^{1}$ then $f(d)$ is a subset of some link of $C^{1}$.

Let

$$
D^{i}=d_{1}^{i}, d_{2}^{i}, \ldots, d_{k_{i}}^{i} \quad \text { with } P \in d_{1}^{i}, Q \in d_{k_{i}}^{i},
$$

and

$$
C^{i}=c_{1}^{i}, c_{2}^{i}, \ldots, c_{l_{i}}^{i} \quad \text { with } f(P) \in c_{1}^{i}, f(Q) \in c_{l_{i}}^{i} .
$$

If $i \in\left\{1, \ldots, k_{1}\right\}$, let $N^{1}(i)$ denote the first integer $n$ so that $f\left(d_{i}^{1}\right) \subset c_{n}^{1}$. Since $C^{1}$ and $D^{1}$ are chains from $f(P)$ to $f(Q)$ and $P$ to $Q$ respectively, then $N^{1}$ is a pattern and $N^{1}(1)=1, N^{1}\left(k_{1}\right)=l_{1}$.

Let $\delta_{2}$ be a positive number less than the distance between every two nonintersecting links of $C^{1}$ and less than $\delta_{1}$. Let $B^{1}=C^{1}$.

There is an integer $j_{2}$ and a consolidation $B^{2}$ of the links of $C^{j_{2}}$ so that $B^{2}$ is a chain from $f(P)$ to $f(Q)$ that follows $N^{1}$ in $C^{1}$. From the definition of consolidation 
it follows that the closure of two links of $B^{2}$ intersect only if the links themselves do so. Let $0<\delta_{3}<\delta_{2}$, so that the distance between nonintersecting links of $B^{2}$ is at least $\delta_{3}$ and let $C^{j_{3}}$ be a $\delta_{3} / 3^{3}$ chain which refines $B^{2}$ so that at least ten links of $C^{j_{3}}$ are needed to connect two nonintersecting links of $B^{2}$, and let $N^{2}$ be a pattern of $C^{j_{3}}$ in $B^{2}$. Let $B^{3}=C^{j_{3}}$.

Let $A^{1}=D^{1}$. There is an integer $i_{3}>1$ and a chain $A^{2}$ from $P$ to $Q$ that is a consolidation of $D^{i_{3}}$ that follows $N^{2}$ in $D^{1}$. Let $0<\delta_{4}<\delta_{3}$, let $D^{i_{4}}$ be a $\delta_{4} / 3^{4}$ chain which refines $A^{2}$, and let $N^{3}$ be a pattern of $D^{i_{4}}$ in $A^{2}$. Let $A^{3} \doteq D^{i_{4}}$. There is an integer $j_{4}$ and a chain $B^{4}$ from $f(P)$ to $f(Q)$ that is a consolidation of $C^{j_{4}}$ so that $B^{4}$ follows $N^{3}$ in $B^{3}$. Let $\delta_{5}$ be a positive number, $\delta_{5}<\delta_{4}$, so that the distance between nonintersecting links of $B^{4}$ is at least $\delta_{5}$.

Thus by induction we can construct a sequence $A^{1}, A^{2}, \ldots$ of chains from $P$ to $Q$ covering $X$, a sequence $B^{1}, B^{2}, \ldots$ of chains from $f(P)$ to $f(Q)$ covering $\tilde{X}$, a decreasing sequence $\delta_{1}, \delta_{2}, \ldots$ of positive numbers so that if $k$ is an even positive integer:

(i) the distance between nonintersecting links of $B^{k}$ is at least $\delta_{k+1}$,

(ii) $B^{k+1}=C^{j_{k+1}}$ is a $\delta_{k+1} / 3^{k+1}$ chain which refines $B^{k}$ so that at least ten links of $B^{k+1}$ are needed to connect two nonintersecting links of $B^{k}$,

(iii) $B^{k+1}$ follows pattern $N^{k}$ in $B^{k}$,

(iv) $A^{k+1}$ follows pattern $N^{k+1}$ in $A^{k}$,

(v) $A^{k+2}$ is a consolidation of $D^{j_{k+3}}$ which is a $\delta_{k+2} / 3^{k+2}$ chain which refines $A^{k+2}$ and follows pattern $N^{k+2}$ in $A^{k+1}$, and

(vi) $B^{k+2}$ follows pattern $N^{k+1}$ in $B^{k+1}$.

Note that $B^{k+1}$ and $A^{k}$ have the same number of links. Also, for $k$ even, $A^{k-1}$ and $A^{k}$ are $\delta_{k} / 3^{k}$ chains and $B^{k+1}$ and $B^{k+2}$ are $\delta_{k+1} / 3^{k+1}$ chains, and if $k$ is an even integer then two nonintersecting links of $B^{k}$ are at least $\delta_{k+1}$ apart.

We have now defined a sequence of chains which satisfy the hypothesis of Theorem B. So there exists a homeomorphism $h$ mapping $X$ into $\tilde{X}$. We shall outline the construction of the homeomorphism and state some of its properties which the reader may verify by consulting Bing's proof of Theorem $B$.

Suppose $x \in X$. Then let $r_{i}^{x}$ denote an integer $n$ so that $x \in a_{n}^{i}$. Thus $x=$ $\bigcap_{i=1}^{\infty} a_{r_{i}^{x}}^{i}$. For each positive integer $i$ let $P_{r_{i}^{x}}^{i+1}$ be a point in $b_{r_{i}^{x}}^{i+1}$. It follows from the construction that $\left\{P_{r_{i}^{x}}^{i+1}\right\}_{i=1}^{\infty}$ is a Cauchy sequence. Also since $\lim _{i \rightarrow \infty}$ mesh $B^{i}=0$ it follows that if $Q_{r_{i}^{x}}^{i+1} \in b_{r_{i}^{x}}^{i+1}$, then $\left\{Q_{r_{i}^{x}}^{i+1}\right\}_{i=1}^{\infty}$ has the same sequential limit as $\left\{P_{r_{i}^{x}}^{i+1}\right\}_{i=1}^{\infty}$. Thus if $h(x)=\lim _{i \rightarrow \infty} P_{r_{i}^{x}}^{i+1}$, then $h$ is well defined and is the required transformation. Further we have that

$$
\lim _{i \rightarrow \infty} d\left(h(x) \cdot b_{r_{i}^{x}}^{i+1}\right)=0
$$

and in particular $d\left(h(x), b_{r_{1}^{r}}^{2}\right)<\delta_{1}<\varepsilon / 4$.

The fact that $h$ is a homeomorphism of $X$ into $\tilde{X}$ follows from condition (*) together with the ten-link condition (condition (ii)). For each positive integer $k$. $A^{k}$ is a chain from $P$ to $Q$ and $B^{k}$ is a chain from $f(P)$ to $f(Q)$. Thus $h(P)=f(P)$ and $h(Q)=f(Q)$ and hence $h$ is onto.

We now need to prove the following claim.

ClaIM. For each $x \in X, d(f(x), h(x))<\varepsilon$. 
Proof. Suppose $x$ is a point of $X$ and $x \in d_{i}^{1}$. Then $f(x) \in f\left(d_{i}^{1}\right)$ and $f\left(d_{i}^{1}\right) \subset c_{N(i)}^{1}$. Let $x \in a_{r_{1}^{x}}^{1}$, and since $A^{1}=D^{1}, r_{1}^{x}$ is one of $i-1, i$, or $i+1$. So $N^{1}\left(r_{1}^{x}\right)$ is one of $N(i-1), N(i)$, or $N(i+1)$. Now $B^{2}$ follows $N^{1}$ in $C^{1}$ so

$$
b_{r_{1}^{x}}^{2} \subset c_{N^{1}\left(r_{1}^{x}\right)}^{1} \subset c_{N(i-1)}^{1} \cup c_{N(i)}^{1} \cup c_{N(i+1)}^{1} .
$$

Thus, from above,

$$
d\left(b_{r_{1}^{x}}^{2}, h(x)\right)<\frac{\varepsilon}{4}, \quad d\left(h(x), c_{N(i)}^{1}<2 \frac{\varepsilon}{4}=\frac{\varepsilon}{2} .\right.
$$

So $d(h(x), f(x))<\varepsilon / 2+\varepsilon / 4<\varepsilon$. This proves the theorem.

Comment. Recently Wayne Lewis has announced similar results.

\section{REFERENCES}

[B] R. H. Bing, A homogeneous indecomposable plane continuum, Duke Math. J. 15 (1948), 729-742.

[Br1] B. Brechner, Homeomorphism groups of chainable and homogeneous continua, Topology Proc. (to appear).

[Br2] (1966), 516-548.

[Lw] W. Lewis, Pseudo-arcs and connectedness in homeomorphism groups, Proc. Amer. Math. Soc. 87 (1983), 745-748.

[M] R. L. Moore, Foundations of point set theory, Amer. Math. Soc. Colloq. Publ., vol. 13, Providence, R. I., 1962.

Department of Mathematics, Auburn University, Auburn, Alabama 36849 